\title{
Formalization of Emotions with Intensity
}

\author{
Rui Qiao \\ Wuhan University of Science and Technology \\ Wuhan, China \\ Email:freeqr@gmail.com
}

\author{
Heng $\mathrm{He}$ \\ Wuhan University of Science and Technology \\ Huazhong University of Science and Technology \\ Wuhan, China \\ Email:willam1981@gmail.com
}

\begin{abstract}
Logical formalization of emotions moves forward and develops more widely accepted and clearly defined emotion models. However, there is little quantitative analysis of emotions based on modal logic. We put forward a logic LEI to represent quantitative belief, preference and disgust. Then, event-based emotions, agent-based emotions in OCC model and their intensity are formalized by the logic.
\end{abstract}

Keywords-Emotion with intensity; LEI; quantitative

\section{INTRODUCTION}

Discrete theories, continuous theories, physiological theories, and cognitive theories constitute main trends in psychological research on emotions [8]. The OCC theory[3] is one of appraisal theories as a branch of cognitive theories[8].

An important aspect of emotion simulation is the way in which changes in emotion eliciting situations can give rise to different intensities in resulting emotion instances[9]. Literature[9] uses the work of Ortony, et al[3] to propose a set of emotion intensity variables to be used in modeling the causes of varying emotion intensity.

Logical formalization is a well-defined scientific program to move forward and develop more widely accepted and clearly defined models[7]. Much work models emotions based on modal logics and cognitive theories, such as Adam[7],[8], Steunebrink[20], [6], Meyer[13], Pereira[10], Nguyen[17], Ochs[18], and $\mathrm{Hu}[14]$. However, there is little quantitative analysis of emotions based on modal logic. The papers[11],[12] formalized expectationbased emotions and their intensity. Steunebrink[5], [4], [20] combine the qualitative and quantitative aspects of emotions, and formalize action tendency based on changing emotion intensity.

In this paper, we put forward a logic LEI to present quantitative belief, preference, and disgust. Then, eventbased emotions, agent-based emotions in OCC model [3] and their intensity are formalized by the logic.

The rest of the paper is organized as follows: Section2 presents the logic LEI and some abbreviations. Section3 formalizes emotions with intensity. Section 4 compares this paper with others' work.

\section{LOGIC LEI}

A. Syntax

Let $R$ denote the real numbers set, $R^{+}$denote non- negative real number set, $R^{-}$denote non-positive real number set, $[0,1],(0,1],[0,1),(0,1)$ denote 0,1 closed interval, two 0,1 half open half closed interval, open interval respectively.

Definition 1(Language) Assume three countable sets: a set of agents, denoted as $\mathrm{Ag}$; a set of atomic propositions denoted as $\mathrm{P}$; a set of atomic actions denoted as $A c$. The language $L_{L E I}$ of LEI is defined by the BNF grammar:

atomic formulas: $p\left|b e_{i_{0}}^{k}\right| p d_{i_{0}}^{h}$

formulas: $\neg \varphi|\varphi \vee \phi| G \varphi|H \varphi|[i: \alpha] \varphi|\varphi[i: \alpha]| E \varphi$

Where $p \in \mathrm{P}, \alpha \in A c, i_{0} \in A g, i \in A g, k \in[0,1], h \in R$

$b e_{i_{0}}^{k}$ can be read "The given situation has a degree of belief $k$ according to agent $i_{0}$ at the current situation". $p d_{i_{0}}^{h}\left(h \in R^{+}\right)$can be read "The given situation has a degree of preference $h$ according to agent $i_{0}$ at the current situation". $p d_{i_{0}}^{h}\left(h \in R^{-}\right)$can be read "The given situation has a degree of disgust $-h$ according to agent $i_{0}$ at the current situation". $G \varphi$ reads"henceforth, " $\varphi$ is true." $H \varphi$ reads " $\varphi$ has always been true in the past." $[i: \alpha] \varphi$ reads "After agent $i$ executes action $\alpha, \varphi$ holds surely." $\varphi[i: \alpha]$ reads "Before agent $i$ executes action $\alpha, \varphi$ holds surely." $E \varphi$ expresses that $\varphi$ is possibly true.

\section{B. Semantics}

Definition 2 (Model) Given three countable sets: a set of agents, denoted as $\mathrm{Ag}$; a set of atomic propositions denoted as $\mathrm{P}$; a set of atomic actions denoted as $A c$. The model is a 7-tuple

$M_{L E I}=<W, \pi_{i}, \tau_{i}, \mathrm{~T}, \mathrm{~A}, V>$, abbreviated as $M$,

where:

- $W$ : a nonempty set of situations. A situation $w \in W$ is a pair $\langle r, s\rangle$, where $r$ refers to a possible world, and $s$ is a temporal state in the world $r$.

- $\pi_{i}: W \times W \rightarrow[0,1]$ is a function from the Cartesian product on the set of possible situations into closed interval $[0,1]$. 
$-\tau_{i}: W \times W \rightarrow R$ is a function from the Cartesian product on the set of possible situations into the set $R$.

$-\mathrm{T} \subseteq W \times W$ the temporal accessibility relation. It satisfies that $\left(<r_{i}, s_{j}>,<r_{k}, s_{l}>\right) \in \mathrm{T}$ implies $r_{i}=r_{k}$.

Let $P(A)$ denotes the powerset of set $A$.

- A : $A g \times A c \rightarrow P(W \times W)$ associates each action $\alpha \in A c$ of agent $i \in A g$ with the set $\mathrm{A}(i: \alpha) \subseteq \mathrm{T}$ of sequence of two possible situations.

- $V: \mathrm{P} \rightarrow P(W)$ associates each atomic formula into subset of $W$.

Following [10] and [16], let $w_{1}=<r_{1}, s_{1}>$, $w_{2}=<r_{2}, s_{2}>$ be two situations. When $r_{1}=r_{2}, w_{1}, w_{2}$ represent situations in the same world but perhaps different time. On the contrary, when $s_{1}=s_{2}, w_{1}, w_{2}$ represent different possible worlds at the same time.

The function $\pi_{i}$ represents a belief grading of the possible situations, which allows to model the notion of belief with degree. $\pi_{i}(v, w)=\theta$ means: The belief degree of the given situation $w$ is $\theta$ according to agent $i$ at situation $v$. Notice that the belief degree of $w$ according to agent $i$ is affected by the situation $v$ where is the agent $i$.

The function $\pi_{i}$ satisfies $\sum_{w \in W} \pi_{i}(v, w)=1$, which represents the possible situations in $W$ are mutually exclusive and exhaustive. $\pi_{i}(v, w)=0$ means agent $i$ at situation $v$ thinks the current situation cannot be $w$ at all. On the contrary, $\pi_{i}(v, w)=1$ means agent $i$ at situation $v$ thinks the current situation is surely $w \cdot \pi_{i}\left(v, w_{1}\right)>\pi_{i}\left(v, w_{2}\right)$ means at situation $v$, agent $i$ think the current situation is more likely to be the given situation $w_{1}$ than $w_{2}$.

Let $w_{1}=<r_{1}, s_{1}>, w_{2}=<r_{2}, s_{2}>$ be any two situations. We limit $s_{1}=s_{2}$ in $\pi_{i}\left(<r_{1}, s_{1}>,<r_{2}, s_{2}>\right)$. That's to say, we only consider the form $\pi_{i}\left(<r_{1}, s>,<r_{2}, s>\right)$, which characterizes the belief degree of the situation $<r_{2}, s_{2}>$ according to agent $\mathrm{i}$ in the situation $<r_{1}, s_{1}>$ at the same time. The value of $\pi_{i}\left(<r_{1}, s>,<r_{2}, s>\right)$ changes with the temporal state $S$.

Example1 At the situation 1, a monochromatism $i$ takes a face up poker card whose number is $\mathrm{K}$. The belief degrees (noted as $k$ ) of the Hearts $\mathrm{K}$ and spades $\mathrm{K}$ satisfy $k>0$, while the belief degrees of square piece $K$, plum $\mathrm{K}$ and other letters are 0 . At the situation 2, if the monochromatism $i$ takes a card $\mathrm{Q}$, then the belief degrees (noted as $k$ ) of hearts Q and spades Q satisfy $k>0$, while the belief degrees of square piece $\mathrm{Q}$, plum $\mathrm{Q}$ and other letters are 0 . Moreover, the sum of belief degrees of full set of poker cards is 1 in both situations. Assume $i$ can distinguish black and red when he is wearing a color-blind glasses in the next time(next temporal state). At this time, if $i$ takes a face up poker card whose number is $\mathrm{K}$, then the belief degree(noted as $k$ ) of only one of the Hearts $\mathrm{K}$ and spades $\mathrm{K}$ satisfy $k>0$, while the belief degrees of all other cards are 0 .

The function $\tau_{i}$ represents degrees of goodness or badness of the possible situations in $W$, which allows to model the notion of preference and disgust. $\tau_{i}(v, w)=\theta(\theta>0)$ means: The preference degree of $w$ is $\theta$ according to agent $i$ at situation $v . \quad \tau_{i}(v, w)=\theta(\theta<0)$ means: The disgust degree of $w$ is - $\theta$ according to agent $i$ at situation $v$.

$\tau_{i}(v, w)=\theta$ means at situation $v$, agent $i$ neither prefer the situation $w$ nor disgust it. $\tau_{i}\left(v, w_{1}\right)>\tau_{i}\left(v, w_{2}\right)>0$ means at situation $v$, agent $i$ more prefers the situation $w_{1}$ than $w_{2}$. Symmetrically, $\tau_{i}\left(v, w_{1}\right)<\tau_{i}\left(v, w_{2}\right)<0$ presents agent $i$ at situation $v$ more disgusts the situation $w_{1}$ than $w_{2}$.

Like $_{\pi_{i}}$, we limit $s_{1}=s_{2}$ in $\tau_{i}\left(<r_{1}, s_{1}>,<r_{2}, s_{2}>\right)$. The value of $\tau_{i}\left(<r_{1}, s>,<r_{2}, s>\right)$ also changes with the temporal state $S$.

Example2 Generally speaking, a person prefers warm wear and disgusts cool clothes in winter, while he prefers cool clothes and disgusts warm wear in summer. The preference and disgust of the person changes with time.

T represents the internal temporal relation of each world $r$. $w \in V(p)$ means that atomic formula $p$ is true at situation $w$. We recursively define truth of a formula $\varphi$ as follows:

Definition 3(Truth of formula) Given a LEI model $M$, two possible situations $w, v \in W$, a formula $\varphi$ is satisfied in $M$ at situations sequence $\langle v, w\rangle$ is defined as follows:

$M,<v, w>\mid=p$ iff $w \in V(p)$

$M,<v, w>\mid=b e_{i_{0}}^{k}$ iff $\pi_{i_{0}}(v, w)=k \quad(k \in[0,1])$

$M,<v, w>\mid=p d_{i_{0}}^{h}$ iff $\tau_{i_{0}}(v, w)=h(h \in R)$

$M,<v, w>\mid=\neg \varphi$ iff not $M,<v, w>\mid=\varphi$

$M,<v, w>\mid=\varphi \vee \phi$ iff $M,<v, w>\mid=\varphi$ or $M,<v, w>\mid=\phi$

$M,<v, w>\mid=G \varphi$ iff $M,<v, w^{\prime}>\mid=\varphi$ for every $w^{\prime} \in W$ such that $\left(w, w^{\prime}\right) \in \mathbf{T}$

$M,<v, w>\mid=H \varphi$ iff $M,<v, w^{\prime}>\mid=\varphi$ for every $w^{\prime} \in W$ such that $\left(w^{\prime}, w\right) \in \mathrm{T}$

$M,<v, w>\mid=[i: \alpha] \varphi$ iff for all $w^{\prime} \in W$ such tha $t\left(w, w^{\prime}\right) \in \mathrm{A}(\mathrm{i}: \alpha)$ and $M,<v, w^{\prime}>\mid=\varphi$

$M,<v, w>\mid=\varphi[i: \alpha]$ iff for all $w^{\prime} \in W$ such tha $t\left(w^{\prime}, w\right) \in \mathrm{A}(\mathrm{i}: \alpha)$ and $M,<v, w^{\prime}>\mid=\varphi$

$M,<v, w>\mid=E \varphi$ iff exists $w^{\prime} \in W$ such tha $t M,<v, w^{\prime}>\mid=\varphi$

$M,<v, w>=\varphi$ means the truth value of formula $\varphi$ in the model $M$ is determined by the situations sequence $\langle v, w>$. It expresses that when agent $i_{0}$ is in the situation $v$, the 
formula $\varphi$ is satisfied in the model $M$ at state $w$. From the definition, we can find $v$ has no function on the truth of formulas except formulas containing $b e_{i_{0}}^{k}$ and $p d_{i_{0}}^{h}$.

Definition 4(Validity) A formula $\varphi$ is valid in LEI model $M$, denoted as $M \mid=\varphi$, if and only if $M,<v, w>\mid=\varphi$ for every $v, w$ in LEI $M . \varphi$ is LEI valid, denoted as $=\varphi$, if and only if $\varphi$ is valid in every LEI model $M$.

Now, we extend belief degree of a possible situation to belief degree of a formula.

Definition 5(Belief degree of a formula) The belief degree of a formula $\varphi$ according to an agent $i$ at the situation $w$ in model $M$, noted $\pi_{i}^{w}(\varphi)$, is defined as: $\pi_{i}^{w}(\varphi)=\sum_{M\left\langle\left\langle w_{i}\right\rangle=\varphi\right.} \pi_{\left(w, w^{\prime}\right)}$

The belief degree of a formula $\varphi$ according to an agent $i$ at the situation $w$ is the sum of $\pi_{i}\left(w, w^{\prime}\right)$ where $<w, w^{\prime}>$ satisfies the formula $\varphi$ in $\operatorname{model} M$.

Since $\quad \pi_{i}\left(w, w^{\prime}\right) \in[0,1] \quad$ and $\quad \sum_{w^{\prime} \in W} \pi_{i}\left(w, w^{\prime}\right)=1 \quad$, $\pi_{i}^{w}(\varphi) \in[0,1]$ holds.

From the definition of $\pi_{i}^{w}(\varphi)$ and properties of function $\pi_{i}$, we get the properties of function $\pi_{i}^{w}$ :

$(\pi \neg) \pi_{i}^{w}(\varphi)+\pi_{i}^{w}(\neg \varphi)=1$

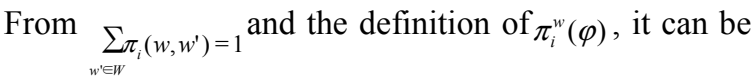
proved easily.

Also, we get the following properties. $(\pi \wedge) \pi_{i}^{\mathrm{w}}(\varphi \wedge \psi) \leqslant \min \left\{\pi_{i}^{w}(\varphi), \pi_{i}^{w}(\psi)\right\}$

Proof: The set $X \subseteq W$ of situations satisfies formula $\varphi$, and the set $Y \subseteq W$ of situations satisfies formula $\psi$. Let $A=X \cap Y$ and $B=X \cup Y$. The situations in $X$ - $A$ satisfy $\varphi$, but do not satisfy $\psi$. Hence they cannot contribute belief degree to $\varphi \wedge \psi$. For the similar reason, situations in $Y$ - $A$ cannot contribute belief degree to $\varphi \wedge \psi$ too. Only situations in $X \cap Y$ contribute belief degree to $\varphi \wedge \psi$. Since $X \cap Y \subseteq X$ and $X \cap Y \subseteq Y$, and for any $w, w^{\prime}, \pi_{i}\left(w, w^{\prime}\right)>0$, we can get $\pi_{\mathrm{i}}^{\mathrm{w}}(\varphi \wedge \psi) \leqslant \min \left\{\pi_{i}^{w}(\varphi), \pi_{i}^{w}(\psi)\right\}$

Symmetrically, we can find:

$(\pi \vee) \pi_{\mathrm{i}}^{\mathrm{w}}(\varphi \vee \psi) \leqslant \max \left\{\pi_{i}^{w}(\varphi), \pi_{i}^{w}(\psi)\right\}$

From $(\pi \wedge)$ and $(\pi \vee)$, we know that the belief degree of $\varphi \wedge \psi$ is smaller than the belief degrees of both $\varphi$ and $\psi$, while the belief degree of $\varphi \vee \psi$ is larger than the belief degrees of both $\varphi$ and $\psi$.

The preference (disgust) degree of formula is extended from the preference (disgust) degree of possible situation.

Definition 6(Preference-Disgust degree of a formula) The preference-disgust degree of a formula $\varphi$ at situation $w$ according to agent $i, \operatorname{noted} \tau_{i}^{w}(\varphi)$, is defined as:

$\tau_{i}^{w}(\varphi)=\sum_{M,\left\langle w, w^{\prime}\right\rangle \mid=\varphi} \tau_{i}\left(w, w^{\prime}\right)$

The preference-disgust degree of a formula $\varphi$ according to an agent $i$ at the situation $w$ is the sum of $\tau_{i}\left(w, w^{\prime}\right)$ where $\left(w, w^{\prime}\right)$ satisfies the formula $\varphi$ in model $M$.

When $\tau_{i}^{w}(\varphi) \geqslant 0$, it represents preference degree, while when $\tau_{i}^{w}(\varphi) \leqslant 0$, it represents disgust degree.

\section{Abbreviations}

This section lists the following syntactic abbreviations. The previous two are as usual.

$$
\begin{aligned}
& \varphi \rightarrow \phi \stackrel{\text { def }}{=} \neg \varphi \vee \phi \\
& \varphi \wedge \phi \stackrel{\text { def }}{=} \neg(\neg \varphi \vee \neg \phi) \\
& B e l_{i}^{k} \varphi \stackrel{\text { def }}{=} \widehat{\sum}_{\sum_{h_{r}}=k} E\left(b e_{i}^{h_{r}} \wedge \varphi\right)(k \in[0,1]) \\
& \operatorname{Bel}_{i}^{k} \varphi \text { reads "Agent } i \text { believes with the degree } k \text { that }
\end{aligned}
$$

$\varphi$ is true". It can be deduced that $M,<v, w>=B e l_{i}^{k} \varphi$ iff $\pi_{i}^{w}(\varphi)=k$ where agent $i$ is in situation $w$. According to agent $i$ at the situation $w, \operatorname{Bel}_{i}^{k} \varphi$ holds in $M$ at $w$ iff the belief degree of the formula $\varphi$ is $k$.

Example3 Suppose there are altogether five situation sequences $\left.\left.\left.\left\{<w, w_{1}\right\rangle,<w, w_{2}>,<w, w_{3}\right\rangle,<w, w_{4}\right\rangle,<w, w_{5}>\right\}$ satisfying $\varphi$ and $b e_{i}^{h_{1}}, b e_{i}^{h_{1}}, b e_{i}^{h_{1}}, b e_{i}^{h_{12}}, b e_{i}^{h_{12}}$ hold on $\left.<w, w_{1}>,<w, w_{2}>,<w, w_{3}>,<w, w_{4}\right\rangle,<w, w_{5}>$ respectively. Then

$B e e_{i}^{k^{k}} \varphi=E\left(b e_{i}^{h_{11}} \wedge \varphi\right) \wedge E\left(b e_{i}^{h_{1}} \wedge \varphi\right) \wedge E\left(b e_{i}^{h_{11}} \wedge \varphi\right) \wedge E\left(b e_{i}^{h_{r_{2}}} \wedge \varphi\right) \wedge E\left(b e_{i}^{h_{12}} \wedge \varphi\right)$ such that $3 \times h_{r 1}+2 \times h_{r 2}=k$

$$
\begin{aligned}
& P D_{i}^{k} \varphi \stackrel{\text { def }}{=} \widehat{\sum}_{h_{r}=k} E\left(p d_{i}^{h_{r}} \wedge \varphi\right)(k \in R) \\
& \operatorname{Pr}_{i}^{k} \varphi \stackrel{\text { def }}{=} P D_{i}^{k} \varphi k \in \mathrm{R}^{+} \\
& \operatorname{Dis}_{i}^{-k} \varphi=P D_{i}^{k} \varphi k \in \mathrm{R}^{-}
\end{aligned}
$$

where $\operatorname{Dis}_{i}^{-\mathrm{k}} \varphi\left(k \in R^{-}\right)$is equivalent to $\operatorname{Dis}_{i}^{\mathrm{k}} \varphi\left(k \in R^{+}\right)$.

$\operatorname{Pr}_{i}^{k} \varphi$ reads " $\varphi$ is preferred with degree $k$ by agent $i$ ".

$M,<v, w>\mid=\operatorname{Pr} e_{i}^{k} \varphi$ iff $\tau_{i}^{w}(\varphi)=k\left(k \in R^{+}\right)$

$D i s_{i}^{k} \varphi$ reads " $\varphi$ is disgusted with degree $k$ by agent $i$ ".

$M,<v, w>=D i s_{i}^{k} \varphi$ iff $\tau_{i}^{w}(\varphi)=-k\left(k \in R^{+}\right)$

$F \varphi \stackrel{\text { def }}{=} \neg G \neg \varphi$

$F \varphi$ reads " $\varphi$ is true or will be true at sometime in the 
future."

$M,<v, w>\mid=F \varphi$ iff

$M,<v, w^{\prime}>=\varphi$ for exists $w^{\prime}$ such that $\left(w, w^{\prime}\right) \in \mathrm{T}$

$P \varphi \stackrel{\text { def }}{=} \neg H \neg \varphi$

$P \varphi$ reads " $\varphi$ is true or was true at sometime in the past."

$M,<v, w>\mid=P \varphi$ iff

$M,<v, w^{\prime}>\mid=\varphi$ for exists $w^{\prime}$ such that $\left(w^{\prime}, w\right) \in \mathrm{T}$

$<i: \alpha>\varphi \stackrel{\text { def }}{=} \neg[i: \alpha] \neg \varphi$

$<i: \alpha>\varphi$ reads " After agent $i$ executes action $\alpha, \varphi$ maybe holds."

$M,<v, w>\mid=<i: \alpha>\varphi$ iff

exists $w^{\prime}$ such that $\left(w, w^{\prime}\right) \in \mathrm{A}(i, \alpha)$ and $M,<v, w^{\prime}>\mid=\varphi$

$\varphi<i: \alpha>\stackrel{\text { def }}{=} \neg(\neg \varphi[i: \alpha])$

$\varphi<i: \alpha>$ reads " Before agent $i$ executes action $\alpha, \varphi$ maybe holds."

$M,<v, w>\mid=\varphi<i: \alpha>$ iff

exists $w^{\prime}$ such that $\left(w^{\prime}, w\right) \in \mathrm{A}(i, \alpha)$ and $M,<v, w^{\prime}>\mid=\varphi$

$\operatorname{Goal}_{i}^{k} \varphi \stackrel{\text { def }}{=} \operatorname{Pr} e_{i}^{k} F \varphi\left(\mathrm{k} \in \mathrm{R}^{+}\right)$

Goal $_{i}^{k} \varphi$ reads "Agent $i$ takes $\varphi$ as a goal with desirability degree $k$ ".

AntiGoal $_{i}^{k} \varphi \stackrel{\text { def }}{=} \operatorname{Dis}_{i}^{k} F \varphi\left(\mathrm{k} \in \mathrm{R}^{+}\right)$

AntiGoal $_{i}^{k} \varphi$ reads "Agent $i$ takes $\varphi$ as a anti-goal with undesirability degree $k$ ".

\section{EMOTIONS WITH INTENSITY}

Emotions and their intensities are analyzed in the section by the modal operators defined above.

Article [9] proposed three categories of emotion intensity variables. Among them, the stable disposition variables group contains variables that help to determine an agent's bias toward interpreting an emotion-eliciting situation one way or another. The specification stable refers to the position these variables have in determining the relatively stable personalities and roles of the automated agents in simulation. This is not to say that these values cannot change over time, but rather that such changes will be considered moderately permanent, with no tendency to return to the original state. The stable disposition variables group includes two subgroups: appraisal bias variables, stable relationship variables.

Here we only consider appraisal bias variables. They are based on the central intensity variables of Ortony, et al [3], appear as part of the agents' appraisal mechanisms and represent the degrees to which various events, actions of agents, and objects are important to them[9]. The notions of consequence of event, action of agent, and aspect of object are used to distinguish three main categories of emotion types.

In $\operatorname{Emotion}_{i}^{k} \varphi, \quad k\left(k \in R^{+}\right)$is the value of a function taking appraisal bias variables and belief degree variables as function arguments, where Emotion denotes any emotion listed in the following.

\section{A. Event-based Emotion}

If an agent focuses on a consequence of an event, it can appraise this consequence as desirable or undesirable with respect to its' goals. There are two variables to mark desirable or undesirable level.

- Importance to agent $i$ of achieving goal, denoted as $k\left(k \in R^{+}\right)$in expression Goal $_{i}^{k} \varphi$. The value $k$ shows how desirable the achievement of a particular goal $\varphi$ is for the agent $i$.

- Importance to agent $i$ of not having goal blocked, denoted as $k\left(k \in R^{+}\right)$in expression AntiGoal $_{i}^{k} \varphi$. The value $k$ shows how undesirable the blockage of a particular goal $\neg \varphi$ is for the agent $i$.

1) Well-being Emotions: An agent $i$ feels joy (distress) when he is fully convinced an event $\varphi$ as the target(antitarget).

$$
\begin{aligned}
& \operatorname{Joy}_{i}^{k} \varphi \stackrel{\text { def }}{=} \operatorname{Bel}_{i}^{1} \varphi \wedge \text { Goal }_{i}^{m} \varphi\left(m \in R^{+}\right) \\
& \text {Distress }_{i}^{k} \varphi \stackrel{\text { def }}{=} \operatorname{Bel}_{i}^{1} \varphi \wedge \text { AntiGoal }_{i}^{m} \varphi\left(m \in R^{+}\right)
\end{aligned}
$$

The degree $k$ of joy (distress) increases with the desirability(undesirability) degree $m$ of the target (antitarget).

2) Prospect-based Emotions: An agent $i$ feels hope (fear) when he believes a event $\varphi$ as the target(anti-target) to a certain extent.

$$
\begin{aligned}
& \operatorname{Hope}_{i}^{k} \varphi \stackrel{\text { def }}{=} \text { Bel }_{i}^{h} \varphi \wedge \text { Goal }_{i}^{m} \varphi\left(h \in[0,1), m \in R^{+}\right) \\
& \text {Fear }_{i}^{k} \varphi \stackrel{\text { def }}{=} \text { Bel }_{i}^{h} \varphi \wedge \text { AntiGoal }_{i}^{m} \varphi\left(h \in[0,1), m \in R^{+}\right)
\end{aligned}
$$

The degree $k$ of hope (fear) increases with both the belief degree $h$ and the desirability(undesirability) degree $\mathrm{m}$ of the target(anti-target). If agent $i$ does not believe event $\varphi$ happens at all, then it has no hope or fear about $\varphi$. Thus in both definitions of Hope and Fear, if $h=0$, then $k=0$.

Notice that only agent $i$ believes goal $\varphi$ surely, it can feel joy about $\varphi$, otherwise, it just hopes $\varphi$. Similarly, only agent $i$ believes $\varphi$ surely which is the contrary of its'goal, it can feel distress about $\varphi$, otherwise, it just fears $\varphi$.

The agent $i$ feels satisfaction(fearconfirmed) if he is confirmed about the prospect of an event $\varphi$ as target(antitarget).

$$
\text { Satisfaction }_{i}^{k} \varphi \stackrel{\text { def }}{=} \operatorname{Bel}_{i}^{1} \operatorname{PBel}_{i}^{q} \varphi \wedge \operatorname{Goal}_{i}^{m} \varphi \wedge \operatorname{Bel}_{i}^{1} \varphi\left(q \in[0,1), m \in R^{+}\right)
$$


FearConfirmed $_{i}^{k} \varphi \stackrel{\text { def }}{=}$ Bel $_{i}^{1} P$ Bel $_{i}^{q} \varphi \wedge$ AntiGoal $_{i}^{m} \varphi \wedge$ Bel $_{i}^{1} \varphi$

$\left(q \in[0,1), m \in R^{+}\right)$

The degree $k$ of satisfaction(fearconfirmed) increases with the desirability(undesirability) degree $m$ of the target(antitarget). The lower was the belief degree $q$ about the event $\varphi$ according to the agent $i$ in the past, the higher is the degree $k$ of satisfaction(fearconfirmed) after agent $i$ is confirmed about the event $\varphi$. In the past, agent $i$ ever believed $\varphi$ with a certain degree, but it was not sure about $\varphi$. Hence $q<1$ holds.

The agent $i$ feels relief(disappointment) if he is disconfirmed about the prospect of an event $\neg \varphi$ as target(anti-target).

$$
\begin{aligned}
& \operatorname{Re} \operatorname{lief}_{i}^{k} \varphi \stackrel{\text { def }}{=} \operatorname{Bel}_{i}^{1} P B e l_{i}^{q} \varphi \wedge \operatorname{Goal}_{i}^{m} \varphi \wedge \operatorname{Bel}_{i}^{1} \varphi\left(q \in(0,1], m \in R^{+}\right) \\
& \text {Disappoint } \text { ment }_{i}^{k} \varphi \stackrel{\text { def }}{=} \text { Bel }_{i}^{1} P B e l_{i}^{q} \neg \varphi \wedge \text { AntiGoal }_{i}^{m} \varphi \wedge \text { Bel }_{i}^{1} \varphi \\
& \left(q \in(0,1], m \in R^{+}\right)
\end{aligned}
$$

The degree $k$ of relief(disappointment) increases with the desirability(undesirability) degree $m$ of the target(antitarget). The higher is the belief degree $q$ about the event $\varphi$ in the past according to the agent $i$, the higher is the degree $k$ of relief(disappointment) after agent $i$ is disconfirmed about the event. In the past, agent $i$ ever believed $\neg \varphi$ with a certain degree, and it couldn't believe $\varphi$ surely. Hence $q>0$ holds.

If the agent $i$ has no desirability(undesirability) on $\varphi$, then his any of the six prospected-based emotions cannot be aroused when he is confirmed about $\varphi$. Thus in the definitions of the six emotions, if $m=0$, then $k=0$.

3) Fortunes of others Emotions: Agent $i$ feels happy (sorry) for agent $j$ if he believes an event $\varphi$ assumed to be target(anti-target) for the agent $j$, and believing the event $\varphi$ by agent $j$ is the target(anti-target) of agent $i$.

$$
\begin{aligned}
& \text { Happyfor }_{i, j}^{k} \varphi \stackrel{\text { def }}{=} \text { Bel }_{i}^{1} \varphi \wedge \text { Bel }_{i}^{1} \text { Goal }_{j}^{m} \varphi \wedge \text { Goal }_{i}^{n} \text { Bel }_{j}^{1} \varphi\left(m \in R^{+}, n \in R^{+}\right) \\
& \text {Sorryfor }_{i, j}^{k} \varphi=\text { Bel }_{i}^{1} \varphi \wedge \text { Bel }_{i}^{1} \text { AntiGoal }_{j}^{m} \varphi \wedge \text { AntiGoal }_{i}^{n} \text { Bel }_{j}^{1} \varphi \\
& \left(m \in R^{+}, n \in R^{+}\right)
\end{aligned}
$$

The degree $k$ of happyfor(sorryfor) increases with the agent $j$ 's desirability(undesirability) degree $m$ on target(anti-target) $\varphi$, and the agent $i$ 's desirability (undesirability) degree $n$ on target(anti-target) $\mathrm{Bel}_{j}^{1} \varphi$.

Agent $i$ feels resentment(gloating) for agent $j$ if he believes an event $\varphi$ assumed to be target(anti-target) for the agent $j$, and the event $\varphi$ being confirmed by agent $j$ is the anti-target(target) of agent $i$.

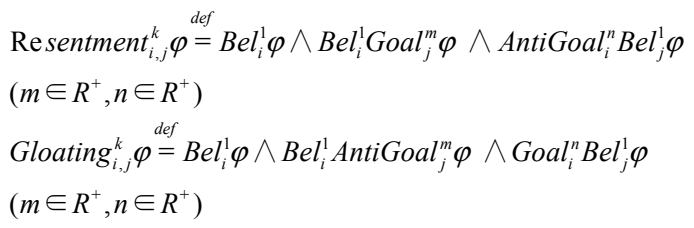

The degree $k$ of resentment(gloating) increases with the agent $j$ 's desirability(undesirability) degree $m$ on target(antitarget) $\varphi$, and the agent $i$ 's undesirability (desirability) degree $m$ on anti-target(target) $\operatorname{Bel}_{i}^{1} \varphi$.

If the agent $j$ has no desirability(undesirability) on $\varphi$, the agent $i$ 's any of the four fortunes of others emotions cannot be aroused when $i$ is confirmed about $\varphi$. Moreover, if the agent $i$ does not care whether $\varphi$ holds for $j$, none of the four emotions can be aroused. Thus in the definitions of the four emotions, if $m=0$ or $n=0$ then $k=0$

\section{B. Agent-based Emotion}

If an agent focuses on an action of another(perhaps itself), he can appraise this action as praiseworthy or blameworthy with respect to his standards. Two variables are used to mark praiseworthy(blameworthy)level.

- Importance to agent $i$ of having standard upheld, denoted as $k$ in $\operatorname{Pr} e_{i}^{k} \varphi$. The value shows how praiseworthy the act of upholding a particular standard $\varphi$ is for the agent $i$

- Importance to agent $i$ of not having standard violated, denoted as $k$ in $\operatorname{Dis}_{i}^{k} \varphi$. The value shows how blameworthy the act of violating a particular standard $\neg \varphi$ is for the agent $i$.

1) Attributions Emotions: An agent $i$ feels admiration (reproach) for an agent $j$ if $j$ has performed the action which caused the result $\varphi$. The agent $i$ believes that before the action $\alpha$, he could not predict the result, and he thought it's ideal(not ideal) for $j$ to do the action to achieve $\varphi$. Here, when an agent thinks something ideal or not ideal, he makes a deontic evaluation. One can think of deontic evaluation as reflecting the preferences of a moral authority or law-giver[15].

$$
\begin{aligned}
& \text { Admiration }_{i, j}^{k}(j: \alpha, \varphi) \stackrel{\text { def }^{\prime}}{=} \operatorname{Bel}_{i}^{1}\left(\left(\operatorname{Pr} e_{i}^{q}<j: \alpha>\varphi \wedge \operatorname{Bel}_{i}^{m}[j: \alpha] \neg \varphi\right)<j: \alpha>\right) \\
& \wedge \operatorname{Bel}_{i}^{1} \varphi\left(m \in(0.5,1), q \in R^{+}\right) \\
& \operatorname{Re} \operatorname{proach}_{i, j}^{k}(j: \alpha, \varphi) \stackrel{\text { def }^{\prime}}{=} \operatorname{Bel}_{i}^{1}\left(\left(\operatorname{Dis}_{i}^{q}<j: \alpha>\varphi \wedge \operatorname{Bel}_{i}^{m}[j: \alpha] \neg \varphi\right)<j: \alpha>\right) \\
& \wedge \operatorname{Bel}_{i}^{1} \varphi\left(m \in(0.5,1), q \in R^{+}\right)
\end{aligned}
$$


The degree $k$ of admiration (reproach) increases with the agent $j$ 's preference(disgust) degree $q$ on result $\varphi$ from the action $\alpha$, and the agent $i$ 's belief degree $m$ on the opposite result $\neg \varphi$. If the agent $i$ has no preference(disgust) on the result $\varphi$ caused by the agent $j$ 's action $\alpha, i$ 's emotions Admiration and Reproach cannot be stimulated when he is confirmed about $\varphi$. Thus in the definitions of the two emotions, if $q=0$ then $k=0 . m>0.5$ means that before executing action $\alpha$, agent $i$ more convinced the action resulting in $\neg \varphi$ than $\varphi$.

When the agent $i$ and $j$ are the same agent, the two emotions above are known exactly as the two emotions in the following.

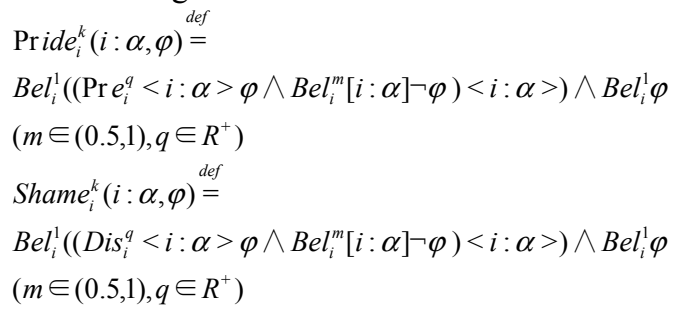

2) Compound Emotions: Some emotions can be composed by the above-mentioned emotions.

$$
\begin{aligned}
& \operatorname{Gratification}_{i}^{k}(i: \alpha, \varphi) \stackrel{\text { def }}{=} \operatorname{Pr} i d e_{i}^{h}(i: \alpha, \varphi) \wedge \operatorname{Joy}_{i}^{q} \varphi \\
& \operatorname{Re} \operatorname{morse}_{i}^{k}(i: \alpha, \varphi) \stackrel{\text { def }}{=} \operatorname{Shame}_{i}^{h}(i: \alpha, \varphi) \wedge \operatorname{Distress}_{i}^{q} \varphi \\
& \operatorname{Gratitude}_{i, j}^{k}(i: \alpha, \varphi) \stackrel{\text { def }}{=} \text { Admiration }_{i, j}^{h}(j: \alpha, \varphi) \wedge \text { Joy }_{i}^{q} \varphi \\
& \text { Anger }_{i, j}^{k}(j: \alpha, \varphi) \stackrel{\text { def }}{=} \operatorname{Re} \operatorname{proach}_{i, j}^{h}(j: \alpha, \varphi) \wedge \operatorname{Distress}_{i}^{q} \varphi
\end{aligned}
$$

$k$ monotonically increases with $h$ and $q$ in the definitions of Gratification, Remorse, Gratitude, Anger.

\section{RELATED WORK}

Articles [1][2] use a fuzzy modal logic to formalize graded believes and desires. The basic idea of the approach is to consider the belief and desire degree of a (classical)proposition as the truth-degree of a fuzzy modal proposition. Probabilistic logic[19] defines the truth value of sentence $S$ to be the probability of $S$. In comparison, we handle the belief, preference-disgust degree of formulas apart from the truth of formulas.

Articles [12],[11] use epistemic equivalence relation $\sim$ on possible worlds to interpret epistemic operator $[K]$. In our models, there's no epistemic equivalence relation $\sim$ on possible situations. Moreover, articles [12],[11] give possible worlds (noted as $w$ ) which are at the same equivalence class as agent's world fixed plausibility grading $\max -\kappa_{\text {exc }}(w) \in R^{+}$, other possible worlds plausibility grading $\max -\kappa_{\text {exc }}(w)=0$. We give possible situations(noted as $w$ ) different belief grading $\pi_{i}(v, w)$ according to the different situations(noted as $v$ ) in which agent $i$ exists. Fixing situation $v$, the $\pi_{i}(v, w)$ of the situations (noted as w) sum to 1 because the sets of possible situations are mutually exclusive and exhaustive.

Paper[21] compared OCFs theory and probability theory. In the theory of OCFs, a proposition may be conceived as a team consisting of its members; in this race, each such team is just as good as its best members. In probability theory, a proposition may also be conceived as a team consisting of its members; but each such team is as weighty and fares as well in this competition as the sum of the masses of its members.

Articles [12],[11] defined

$\max -\kappa_{\text {exc }}(\varphi)=\max -\min \left\{\kappa_{\text {exc }}(v)|M, v|=\varphi\right.$ and $\left.w \sim v\right\}$ as the plausibility degree of a formula $\varphi$ according to agent at world $w$, which followed the theory OCF[21]. Following probability theory, the probability(the probabilistic logic truth value) of any formula $\varphi$ is taken to be the sum of the probabilities of all worlds in which $\varphi$ is true[19]. Our article defines $\pi_{i}^{w}(\varphi)=\sum_{M,\left\langle w, w^{\prime}\right\rangle=\varphi} \pi_{i}\left(w, w^{\prime}\right)$ as the plausibility degree of a formula $\varphi$ according to agent at situation $w$.

As the motivational counterpart of the notion of plausibility degree, articles [12],[11] used $\kappa_{\text {des }}(w)$ and $\kappa_{\text {des }}^{w}(\varphi)$ to define desirability degree of worlds and formulas respectively. Like them, we also give the motivational counterpart of the notion of belief degree denoted as $\tau_{i}(v, w)$ and $\tau_{i}^{w}(\varphi)$.

Articles[12],[11] used $\kappa_{\text {des }}^{w}(\varphi)$ (the range of $\kappa_{\text {des }}^{w}$ is the finite set of natural numbers Num) to interpret the graded goal Goal ${ }^{\hbar} \varphi$. We assign $\tau_{i}^{w}(\varphi) \in R$, where $\tau_{i}^{w}(\varphi) \in R^{+}$ and $\tau_{i}^{w}(\varphi) \in R^{-}$interpret graded preference Pre and graded

disgust Dis respectively.

\section{CONCLUSION}

We have proposed the logic called LEI to represent quantitative belief, preference and disgust. Then we have formalized event-based emotions, agent-based emotions and their intensity by the logic. In future work, we will consider the following aspects. We will give a formal analysis of the object-based emotion. We should also investigate the relation between preference degree of formula $\varphi$ and disgust degree of $\neg \varphi$. Furthermore, properties of the logic LEI will be explored, and implement of emotional agents based on the logical analysis will be considered too.

\section{REFERENCES}


[1] L. A.Casali and C.Sierra. Graded bdi models for agent architectures. In 5th International Workshop on Computational Logic in MultiAgentSystems, pages 126-143. Springer, 2004.

[2] L. A.Casali and C.Sierra. A graded bdi agent model to represent andreason about preferences. Artificial Intelligence, pages 1468$1478,2011$.

[3] A. A.Ortony, G.L.Clore. the cognitive structure of emotions. Cambridge University Press, 1988.

[4] J. M. B.R. Steunebrink, M.Dastani. Towards a quantitative model ofemotions for intelligent agents. In Proceedings of the twentysecond AAAI Conference on Artificial Intelligence, 2007.

[5] J. M. B.R. Steunebrink, M.Dastani. a formal model of emotionbasedaction tendency for intelligent agents. In portuguese conference on artificial intelligence, pages 174-186. Springer, 2009.

[6] J. M. B.R.Steunebrink, M.Dastani. A formal model of emotion triggers:an approach for bdi agents. Synthese, 185, 2012.

[7] D. L. C. Adam, A. Herzig. A logical formalization of the occ theory of emotions. Synthese, 168(2):201-248, 2009.

[8] C.Adam. Emotions:from psychological theories to logical formalization and implementation in a BDI agent. $\mathrm{PhD}$ thesis, 2007.[9] G. C.Elliott. Variables influencing the intensity of simulated affective states. In Proceedings of the AAAI, pages 58-67, 1993.

[10] E. D.Pereira and N.Moreira. Formal modelling of emotions in bdiagents. In 8th International Workshop, Computational Logic in Multi-Agent Systems, pages 62-81. Springer, 2008.

[11] E.Lorini. A dynamic logic of knowledge, graded beliefs and gradedgoals and its application to emotion modelling. In Proceedings of theThird international conference on Logic, rationality, and interaction,pages 165-178. Springer-Verlag, 2011.

[12] E.Lorini. Qualitative and quantitative aspects of expectation-based emotions: a logical approach. 2011.

[13] J.Ch.Meyer. Reasoning about emotional agents. International Journal of Intelligent Systems, pages 601-619, 2006.

[14] M. J.Hu, C.Guan and F.Lin. model of emotional agent. In International Conference on Principles and Practice of Multi-Agent Systems, pages 534-539. Springer, 2006.

[15] J.v.Benthem and F.Liu. Deontic logic and changing preferences.

[16] K.Schild. On the relationship between BDI logics and standard logics of concurrency. Autonomous Agents and Multi-Agent Systems, 3(3):259-283, 2000.

[17] M.H.Nguyen. A Logical Framework for Trust-Related Emotions: Formal and Behavioral Results. PhD thesis, computer sciences / artificialintelligence, University of Toulouse, 2010.

[18] D. M.Ochs, K.Devooght and C.Pelachaud. a computaional model ofcapability-based emotion elicitaion for rational agent. In Proceedings of the 1st workshop on Emotion and Computing-Current Research and Future Impact, 2006.

[19] N. Nilsson. Probabilistic logic. Artificial Intelligence Preprint Series, 1986.

[20] B. Steunebrink. The Logical Structure of Emotions. PhD thesis, Utrecht University, 2010.

[21] W.Spohn. Ordinal conditional functions: a dynamic theory of epistemic states. Causation, coherence, and concepts, 256:19-41, 2008. 